\title{
APROTININ ADMINISTRATION IN THE PERICARDIAL CAVITY DOES NOT PREVENT PLATELET ACTIVATION
}

Kyra N. Maquelin, $\mathrm{MD}^{\mathrm{a}}$ Rienk Nieuwland, $\mathrm{PhD}^{\mathrm{b}}$ Eef G. W. M. Lentjes ${ }^{b}$ Anita N. Böing b

Bas Mochtar, MD, $\mathrm{PhD}^{\mathrm{a}}$

Leòn Eijsman, $\mathrm{MD}, \mathrm{PhD}^{\mathrm{a}}$

Augueste Sturk, $\mathrm{PhD}^{\mathrm{b}}$
Objectives: Aprotinin is frequently administered systemically to patients undergoing cardiopulmonary bypass to inhibit activation of platelets and plasma protein systems and thus reduce postoperative blood loss. Two reports on local aprotinin administration, that is, into the pericardial cavity, also indicated improvement in postoperative blood loss, but the underlying mechanism was not investigated. We previously reported the disappearance of glycoprotein Ib from the platelet surface and the appearance of plateletderived microparticles in the pericardial cavity of patients undergoing cardiopulmonary bypass as signs of platelet activation. Here, we investigated whether such local aprotinin administration reduced platelet activation.

Methods: In a double-blind study, 6 patients received aprotinin (500,000 KIU) into the pericardial cavity during the operation and 7 patients received a placebo. Platelet surface glycoprotein $\mathrm{Ib}$ expression, concentration of microparticles, and concentration of complexes of platelets with leukocytes, erythrocytes, or each other, were measured by flow cytometry.

Results: We confirmed the reduced glycoprotein Ib expression and the increased concentration of microparticles in the pericardial cavity, as previously reported, and found no increased concentration of platelet complexes. However, no differences between aprotinin and placebo treatments were observed in these platelet activation parameters in the pericardial cavity or the systemic circulation.

Conclusion: We conclude that administration of aprotinin into the pericardial cavity during cardiopulmonary bypass and at concentrations similar to the systemic application does not reduce platelet activation in that compartment or the systemic circulation. (J Thorac Cardiovasc Surg 2000;120:552-7)
$E$ xcessive postoperative blood loss due to impaired hemostasis is one of the major complications of heart surgery with cardiopulmonary bypass $(\mathrm{CPB}){ }^{1}$ Until recently, this was thought to be caused by the extensive contact of the patients' blood with the surface

From the Department of Cardiothoracic Surgery, Onze Lieve Vrouwe Gasthuis, ${ }^{\mathrm{a}}$ Amsterdam, and the Department of Clinical Chemistry, Leiden University Medical Center, ${ }^{\mathrm{b}}$ Leiden, The Netherlands.

Received for publication March 13, 2000; revisions requested April 12, 2000; revisions received May 4, 2000; accepted for publication May 8, 2000.

Address for reprints: A. Sturk, PhD, Leiden University Medical Center, Department of Clinical Chemistry, PO Box 9600, 2300 RC Leiden, The Netherlands (E-mail: asturk@lumc.nl).

Copyright (C) 2000 by The American Association for Thoracic Surgery

$0022-5223 / 2000 \$ 12.00+0 \quad \mathbf{1 2 / 1 / 1 0 8 5 3 0}$

doi:10.1067/mtc.2000.108530 of the extracorporeal circuit. This contact was presumed to activate several blood components, including the coagulation system, the fibrinolytic system, the complement system, and platelets. ${ }^{2}$ However, activation of coagulation and fibrinolysis proved to be especially pronounced in the pericardial cavity. ${ }^{3,4}$ In this compartment, blood oozes from damaged vessels and extensively contacts the damaged vessel wall and underlying tissues. It accumulates in the pericardial cavity, from which it is removed by suction and stored in a reservoir. Periodically, the blood from the reservoir is returned into the circulation of the patient. Inhibition of the activation processes in the pericardial cavity could be advantageous. Tatar, ${ }^{5}$ O'Regan, ${ }^{6}$ and their associates demonstrated a reduced postoperative blood loss when aprotinin, a widely known medication to reduce postoperative blood loss if administered systemically, was administered in the pericardial cavity just before the 
sternotomy was closed. However, the underlying mechanism was not investigated. $\mathrm{We}^{7}$ previously reported that the surface expression of glycoprotein Ib (GPIb)an important adhesion receptor of the platelet-was especially reduced in the pericardial cavity during CPB. $\mathrm{We}^{8}$ also reported highly elevated numbers of procoagulant, especially platelet-derived microparticles, in the pericardial cavity.

The aim of the present double-blind placebo-controlled study was to investigate whether the activation of the platelets in the pericardial cavity or the systemic circulation could be reduced by locally administering aprotinin during the operation. The platelet activation parameters included surface expression of GPIb, concentration of platelet-derived microparticles, and platelet complexes as a sign of platelet activation plus their ensuing interaction with other blood cells. ${ }^{9}$

\section{Patients and methods}

Clinical study. This study was approved by the local ethical committee. After their informed consent was obtained, 13 patients undergoing elective coronary artery bypass surgery entered the study. One group of 6 patients received aprotinin (50 mL containing 500,000 KIU, diluted with $50 \mathrm{~mL}$ saline solution to facilitate gradual administration); the other group of 7 patients received a placebo (saline solution) in the pericardial cavity. During CPB, the solutions from the trial bottles were sprinkled with a syringe into the pericardial cavity. The sprinkling was started after the first blood sampling from the pericardial cavity and continued up to the end of CPB, just before administration of protamine sulfate. The randomized, double-blind study medication was supplied by Bayer AG (Leverkusen, Germany). The code was broken after data acquisition was completed. No patient was older than 85 years or had evidence of severe heart failure, renal or hepatic dysfunction, or a bleeding diathesis. No patient was treated with coumarin derivatives, aspirin, dipyridamole, or nonsteroidal anti-inflammatory agents within 5 days before the operation. The study patients did not receive systemic antifibrinolytic agents or aprotinin during CPB. The aprotinin dosage was based on the fact that aprotinin is administered systemically at $2 \times 10^{6} \mathrm{KIU}$ and the assumption that the systemic circulating volume including the extracorporeal circuit would be approximately $6 \mathrm{~L}$ versus the pericardial content of 500 to $1000 \mathrm{~mL}$.

CPB and anesthesia. Anesthesia was induced and maintained with weight-related doses of fentanyl, sufentanil, midazolam, or propofol and pancuronium. The extracorporeal circuit consisted of a Dideco D704 compact-flow system oxygenator (Dideco SpA, Mirandola, Italy) and an S3 roller pump (Stöckert, Munich, Germany). The priming of the extracorporeal circuit contained lactated Ringer's solution (1.3 L), human albumin ( $200 \mathrm{~mL} 20 \%$ weight/volume), mannitol (100 mL 20\% weight/volume), sodium hydrogen carbonate $(50 \mathrm{~mL} 8.4 \%$ weight/volume), heparin $(50 \mathrm{mg})$, and cefamandol (2 g). Each patient received dexamethasone (1 $\mathrm{mg} / \mathrm{kg}$ ). Volume was corrected with lactated Ringer's solution. Heparin (300 IU/kg body weight) was given intravenously before cannulation of the aorta and repeated in a dose of $50 \mathrm{mg}$ whenever the activated clotting time (Hemochron; International Technidyne Corp, Edison, NJ, with kaolin used as an activator) was shorter than $480 \mathrm{sec}-$ onds. Pump flows ranged from 2.0 to $2.4 \mathrm{~L} \cdot \mathrm{m}^{-2} \cdot \mathrm{min}^{-1}$ during hypothermia $\left(28^{\circ} \mathrm{C}-32^{\circ} \mathrm{C}\right)$. Myocardial protection was achieved with modified St Thomas' Hospital solution infused in the aortic root. After decannulation, heparin was neutralized with protamine sulfate at $3 \mathrm{mg} / \mathrm{kg}$ body weight, that is, in a 1:1 ratio of heparin to protamine sulfate.

Blood acquisition. All systemic blood samples were drawn from the same central venous line. The blood samples from the pericardial cavity were taken directly from the cavity with a $10-\mathrm{mL}$ syringe. Sampling points were after induction, before skin incision (sample point 1), 5 minutes after the start of CPB (sample point 2), 10 minutes before release of the aortic crossclamp, at the start of the last distal anastomosis (sample point 3 ), and before protamine administration (sample point 4). Systemic blood samples were taken at sampling points 1 through 4 and samples from the pericardial cavity at points 2 through 4 . Blood samples were taken simultaneously at each sampling point.

Flow cytometric analysis. Whole blood flow cytometry was performed essentially as described by us ${ }^{7}$ with some modifications. Blood was collected in $0.32 \%$ trisodium citrate (final concentration). Within 5 minutes after sampling, 5 $\mu \mathrm{L}$ aliquots of blood were added to tubes containing $35 \mu \mathrm{L}$ of $\mathrm{N}$-2-hydroxyethylpiperazine- $\mathrm{N}$-2-ethanesulfonic acid (HEPES) buffer (sodium chloride, $137 \mathrm{mmol} / \mathrm{L}$; potassium chloride, $2.7 \mathrm{mmol} / \mathrm{L}$; magnesium chloride, $1.0 \mathrm{mmol} / \mathrm{L}$; glucose, 5.6 $\mathrm{mmol} / \mathrm{L}$; HEPES, $20 \mathrm{mmol} / \mathrm{L}$; albumin, $1 \mathrm{mg} / \mathrm{mL}$; sodium phosphate, $3.3 \mathrm{mmol} / \mathrm{L} ; \mathrm{pH} 7.4$ ) and $5 \mu \mathrm{L}$ anti-GPIb (final concentration, $5 \mu \mathrm{g} / \mathrm{mL}$ ). After 15 minutes of incubation at room temperature in the dark, $5 \mu \mathrm{L}$ of 10 -fold diluted phycoerythrin-conjugated streptavidin was added. After another 15 minutes of incubation at room temperature in the dark, 2.5 $\mathrm{mL}$ of HEPES buffer containing $0.2 \%$ paraformaldehyde (weight/volume) was added. No changes in the expression of surface antigens occurred within 48 hours after fixation if platelets were prepared according to this protocol (Berckmans RJ, unpublished results). Anti-GPIb (CLBMB45) was obtained from the Central Laboratory of The Netherlands Red Cross Blood Transfusion Service (Amsterdam, The Netherlands). The antibody is directed against GPIb- $\alpha$. Anti-GPIb was biotinylated as described by Hnatowich, Virzi, and Rusckowski. ${ }^{10}$ Phycoerythrin-conjugated streptavidin was obtained from Dakopatts (Glostrup, Denmark).

The samples were analyzed in a FACScan flow cytometer with Cell-Quest software (Becton Dickinson, San Jose, Calif). Both forward and sideways scatter were set at logarithmic gain. Per sample, a total of 5000 GPIb-positive events (ie, platelets, platelet-derived microparticles, and platelet complexes) were selected by the phycoerythrin-GPIb fluores- 
Table I. Clinical characteristics of the patients

\begin{tabular}{lcc}
\hline & Aprotinin & Placebo \\
\hline Age (y) & $64(55-70)$ & $59(49-75)$ \\
Sex (male/female) & $6: 0$ & $6: 1$ \\
Distal anastomosis (n) & $5(4-6)$ & $4(3-5)$ \\
CPB time (min) & $121(88-188)$ & $101(55-132)$ \\
Crossclamp time (min) & $79(57-126)$ & $64(34-91)$ \\
Blood loss 6 hours postop (mL) & $361(140-900)$ & $345(160-500)$ \\
\hline
\end{tabular}

*The blood loss of the patient in the aprotinin group with a reoperation due to surgical bleeding was not included in the analysis. There were no significant differences between the 2 groups (Mann-Whitney $U$ test, $P>.05$ ).

cence at $585 \mathrm{~nm}$. Platelets (region 2), microparticles (region 1), and complexes (region 3) were identified by their lightscattering characteristics. Platelet surface expression of GPIb was first expressed as the mean fluorescence intensity of the platelet population, which reflects the surface expression of GPIb of the total platelet population. In a second analysis, the percentage of platelets with decreased GPIb expression was evaluated by setting a threshold arbitrarily at $10 \%$ with the first systemic sample of each patient. Thus, subpopulations of platelets with reduced GPIb expression may become apparent. ${ }^{11}$ The absolute concentration of microparticles, that is, corrected for hemodilution, in sample $\mathrm{x}$ was calculated by the following formula: (Platelet count in blood sample $\mathrm{x}$ ) $\times$ ([Percent events in region 1 of blood sample $\mathrm{x}] /[$ Percent events in region 2 of blood sample $\mathrm{x}]) \times([$ Immunoglobulin $\mathrm{G}$ concentration in first systemic sample]/[Immunoglobulin $\mathrm{G}$ concentration in sample $\mathrm{x}]$ ). The concentration of platelet complexes was similarly calculated on the basis of the number of events in region 3. No attempt was made to subdivide those complexes into platelet-platelet, platelet-leukocyte, or platelet-erythrocyte complexes.

Statistical analysis. Data were analyzed with SPSS for Windows software, version 9.0 (SPSS, Inc, Chicago, Ill). Differences between the systemic and the pericardial cavity samples within the placebo and the aprotinin patient groups were evaluated by GLM univariate multiple variation analysis at an overall significance level of $P=.05$, followed by post hoc analysis by means of the Scheffé test. The MannWhitney $U$ test was used to test differences between the placebo and the aprotinin groups first at each individual sample point and subsequently between the systemic samples of those groups at sample points 2 to 4 . It was also used to test between all pericardial samples at those sample points (because the univariate analysis demonstrated no significant differences between sample points 2 to 4 within the patient groups).

\section{Results}

Clinical results. The clinical data are presented in Table I. No significant differences were present between the patient group treated with aprotinin and the placebo group in age, number of distal anastomoses, CPB time, crossclamp time, or blood loss within 6 hours after the operation. We also did not observe a difference in the number of patients receiving packed cells during and after the operation ( 3 patients in each group) or the number of packed cells transfused (7 in each group). No complications occurred during or after the operation except in 1 patient, in the aprotinin group, who required re-exploration because of surgical bleeding.

Platelet GPIb expression. The results of the platelet GPIb expression analyses are presented in Fig 1, $A$ and $B$. As we described previously, the platelet surface expression of GPIb in the blood from the pericardial cavity of the patients treated with placebo was significantly less than that of the first systemic blood sample. This was found both in the analysis of the mean GPIb antigen expression of the platelet population (Fig 1, A: systemic sample 1 vs pericardial cavity sample 2; $P=.04)$ and in the analysis of the percentage of platelets with a reduced surface GPIb expression (Fig 1, $B$ : systemic sample 1 vs pericardial cavity samples 2,3 , and 4; all $P<.001)$. Similar results were obtained in the patients treated with aprotinin. The mean GPIb antigen expression was reduced in pericardial cavity samples 2 and $3(P=.004)$ compared with systemic sample 1 and also systemic sample $2(P=.05)$, and the percentage of platelets with a reduced GPIb antigen expression was increased in pericardial samples 2 and 3 compared with systemic sample $1(P=.001$ and .003 , respectively $)$ and also compared with systemic samples $2(P=.014$ and .029 , respectively) and $3(P=.016$ and .032 , respectively). There were no statistically significant differences in mean platelet GPIb antigen expression between the patient groups treated with aprotinin versus placebo at any systemic or pericardial sample point. Also, the pooling of all data within the 2 groups, pooling of the data of the 4 systemic samples, or pooling of the 3 pericardial cavity samples did not result in statistically significant differences ( $P=.34, .18$, and .59 , respectively). Differences also were not obtained with such analyses of the percentages of platelets with reduced GPIb antigen exposure ( $P=.93, .69$, and .41 , respectively).

Platelet microparticle formation. The results of the platelet microparticle analyses are provided in Fig 1, $C$. Both in the placebo-treated patient group and in the aprotinin-treated group, we observed a significantly increased concentration of platelet-derived microparticles in pericardial cavity sample 4 versus all 4 systemic samples $(P<.001)$. In both patient groups, the concentration of microparticles in pericardial cavity sample 4 was also increased compared with pericardial cavity sample $2(P<.001)$. Also, with the microparticle analysis, we did not obtain statistically significant differ- 

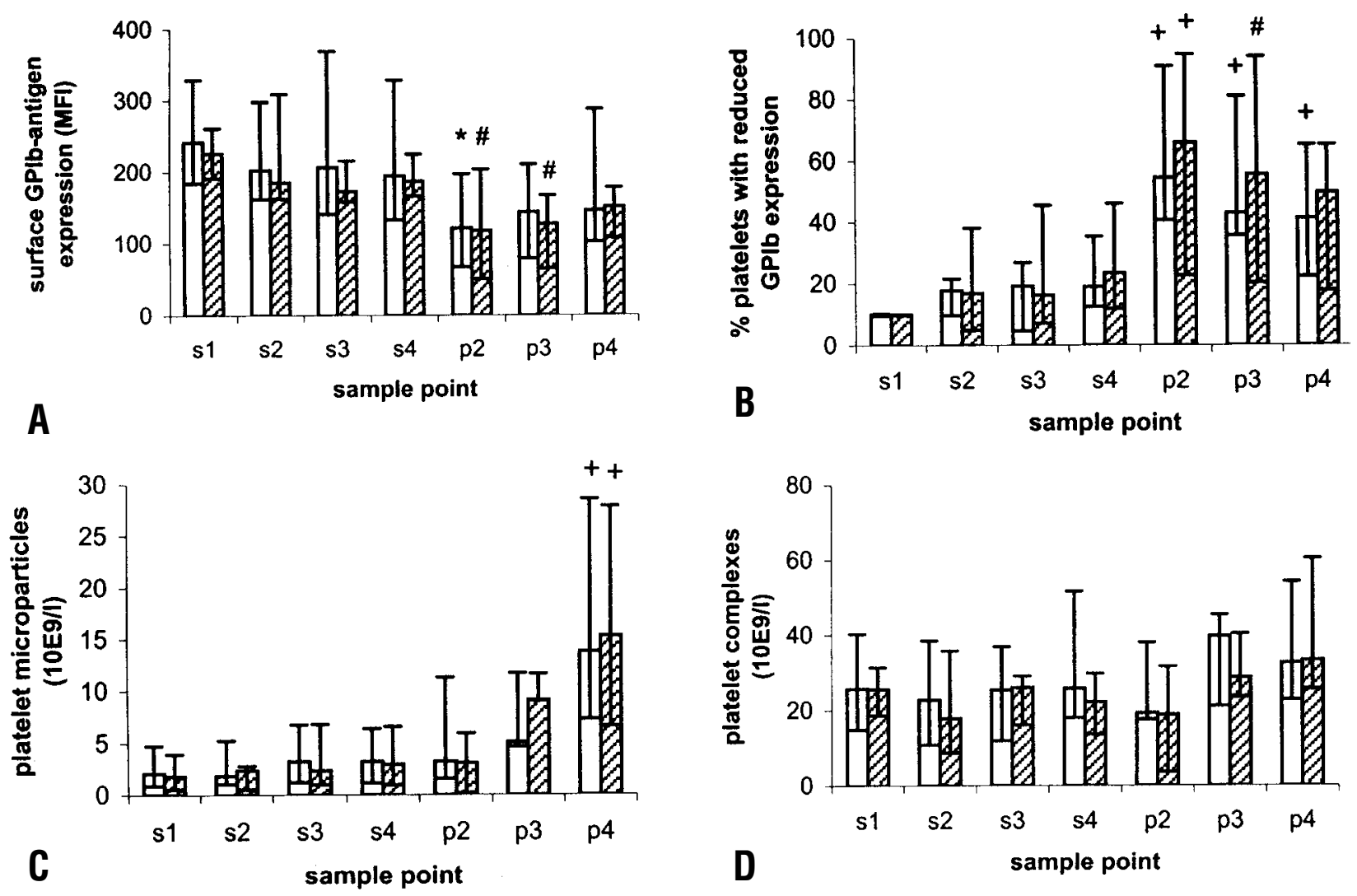

Fig 1. Platelet activation analyses. A, The platelet surface expression of GPIb in the systemic circulation and the pericardial cavity, expressed as the mean fluorescence intensity (MFI), as the mean surface antigen density. B, The percentage of platelets with a reduced expression of GPIb, with the systemic sample at point 1 arbitrarily set at $10 \%$. C, The concentration of platelet-derived microparticles. D, The concentration of platelet complexes, that is, platelet-platelet, platelet-leukocyte, and platelet-erythrocyte complexes. The data are corrected for hemodilution. Open bars represent the placebo group $(\mathrm{n}=7)$ and hatched bars the aprotinin group $(\mathrm{n}=6)$. Median and ranges are provided. ${ }^{*} P<.05,{ }^{\#} P<.005,{ }^{+} P<.001$.

ences in the comparisons of systemic or pericardial cavity samples of placebo- versus aprotinin-treated patient groups at any individual sample point $(P>.05)$, nor did we find this with the data in all systemic plus pericardial samples or solely the systemic or pericardial samples $(P=.35,0.49$, and 0.68 , respectively).

Platelet complexes. The results of the analyses of the platelet complexes are presented in Fig 1, D. No statistically significant differences were detected between any systemic or pericardial cavity samples versus the systemic sample at sample point 1 in the placebo- or aprotinin-treated patient group. Also, the pooling of the data as described above did not result in statistically significant differences between the patient groups at a single sample point $(P>.05)$, in all systemic plus pericardial samples, or in the systemic or pericardial samples separately $(P=.38, .50$, and .75 , respectively).

\section{Discussion}

In the present study, we did not observe differences in platelet activation between the aprotinin- and placebo-treated patient groups by means of the 3 markers used: reduction of platelet surface GPIb expression, microparticle formation, and platelet interaction with each other or other cells. We did not investigate other established parameters to detect platelet activation, for example, surface expression of P-selectin, GP53, or platelet-associated complement-1 binding, because $w^{7}$ previously demonstrated those parameters to be unchanged during CPB in both the systemic circulation and the pericardial cavity.

The clinical relevance of activation of various blood systems in the pericardial cavity was already demonstrated by de Haan and associates, ${ }^{12}$ who found reduced postoperative blood loss if the pericardial 
content was not returned into the patient. However, not returning the content from the pericardial cavity should be balanced against a possible need for blood transfusion, with all its disadvantages. An alternative approach would be to inhibit the activation processes in the pericardial cavity. A widely known medication to prevent blood activation is aprotinin. Systemically administered aprotinin reduces postoperative blood loss. ${ }^{13}$ Tatar, ${ }^{5}$ O'Regan, ${ }^{6}$ and their colleagues demonstrated a reduced postoperative blood loss when aprotinin was administered in the pericardial cavity just before closure of the sternotomy. Bizzarri and coworkers ${ }^{14}$ similarly treated the patients with local aprotinin in addition to administering it systemically. They thereby successfully reduced the postoperative blood loss, but the studies cannot be compared with ours because the local concentration of aprotinin may then be much higher in their studies and will not reduce the intraoperative activation of platelets and plasma protein systems. We attempted to inhibit the platelet activation at an early stage; that is, we administered aprotinin during the operation and at the site presumed to have the highest activation state, the pericardial cavity. ${ }^{7}$

The question could be raised whether the dosage of aprotinin used in the present study was sufficient to prevent platelet activation in the pericardial cavity. The aprotinin dosage used $(500,000 \mathrm{KIU})$ was based on the fact that aprotinin is being administered systemically at a dosage of $2 \times 10^{6} \mathrm{KIU}$ and the assumption that the systemic circulating volume including the extracorporeal circuit would be approximately $6 \mathrm{~L}$ versus the volume of the pericardial content of $500 \mathrm{~mL}$ to maximally $1 \mathrm{~L}$. In vitro, the disappearance of GPIb from the platelet surface can be induced by several stimuli, such as thrombin and plasmin. ${ }^{15,16}$ Aprotinin inhibits plasmin, kallikrein, and thrombin at an inhibition constant $\left(\mathrm{K}_{\mathrm{i}}\right)$ of $0.07 \mathrm{nmol} / \mathrm{L}, 36 \mathrm{nmol} / \mathrm{L}$, and $61,000 \mathrm{nmol} / \mathrm{L}$, respectively. The concentrations used clinically are thus sufficient to inhibit plasmin and kallikrein, but not thrombin. ${ }^{17}$ In the present study, the 500,000 KIU, if presumed to be present in $1 \mathrm{~L}$ of pericardial cavity material, would correspond to $7700 \mathrm{nmol} / \mathrm{L}$. This would be insufficient to inhibit thrombin effectively. If thrombin is indeed causing the platelet activation represented by the disappearance of GPIb from the platelet surface plus microparticle formation, it may be wondered whether sufficiently high dosages of aprotinin could then be obtained.

We did not find a difference in blood loss between the aprotinin- and the placebo-treated patient groups. The question could be raised whether the amount of aprotinin used in the present study was sufficient to expect an effect on blood loss. The aprotinin administered into the pericardial cavity provided a high local concentration, as argued above. Virtually all of it was subsequently given to the patient, because all material from the pericardial cavity was collected into the suction reservoir and returned to the heartlung machine, and the blood in the machine was infused into the patient at the end of the operation. On the one hand, the $0.5 \times 10^{6} \mathrm{KIU}$ of aprotinin thus administered is low compared with the 2 to $6 \times 10^{6}$ KIU regularly used systemically. On the other hand, we administered the aprotinin intraoperatively and thus facilitated continuous inhibition of the activation processes. Tatar, ${ }^{5} \mathrm{O}^{\prime}$ Regan, ${ }^{6}$ and their associates administered the same or twice this dosage of aprotinin at the end of the operation, respectively, and did observe a reduced blood loss, even though the systemic concentration was very low. Viewing these results, we presume that local inhibition is more effective than systemic administration and thus propose that the locally administered high aprotinin concentration is in itself sufficient. The small number of patients in this study may preclude an effect on blood loss. However, we aimed to study the effect of local aprotinin administration on platelet activation and not blood loss.

In conclusion, the administration of 500,000 KIU aprotinin into the pericardial cavity during the $\mathrm{CPB}$ procedure did not inhibit the activation of platelets in this compartment, as measured by reduced surface expression of the GPIb antigen, microparticle formation, and platelet complexes. A reduction in postoperative blood loss or need for blood transfusion is therefore unlikely to be mediated by an effect on the platelet.

\section{REFERENCES}

1. Woodman RC, Harker LA. Bleeding complications associated with cardiopulmonary bypass. Blood 1990;76:1680-97.

2. Edmunds LH Jr. Blood-surface interactions during cardiopulmonary bypass. J Card Surg 1993;8:404-10.

3. Chung JH, Gikakis N, Rao K, Drak TA, Colman RW, Edmunds H Jr. Pericardial blood activates the extrinsic coagulation pathway during clinical cardiopulmonary bypass. Circulation 1996;93:2014-8

4. Tabuchi N, de Haan J, Boonstra PW, van Oeveren W. Activation of fibrinolysis in the pericardial cavity during cardiopulmonary bypass. J Thorac Cardiovasc Surg 1993;106:828-33.

5. Tatar H, Cicek S, Demirkilic U, Ozal E, Suer H, Ozturk O, et al. Topical use of aprotinin in open heart operations. Ann Thorac Surg 1993;55:659-61.

6. O'Regan DJ, Giannopoulos N, Mediratta N, Kendall SWH, Forni A, Pillai R, et al. Topical aprotinin in cardiac operations. Ann Thorac Surg 1994;58:778-81. 
7. Maquelin KN, Berckmans RJ, Nieuwland R, Schaap MCL, ten Have K, Eijsman L, et al. Disappearance of glycoprotein Ib from the platelet surface in pericardial blood during cardiopulmonary bypass. J Thorac Cardiovasc Surg 1998;115:1160-5.

8. Nieuwland R, Berckmans RJ, Rotteveel-Eijkman RC, Maquelin KN, Roozendaal KJ, Jansen PGM, et al. Cell-derived microparticles generated in patients during cardiopulmonary bypass are highly procoagulant. Circulation 1997;96:3534-41.

9. Rinder CS, Gaal D, Student LA, Smith BR. Platelet-leukocyte activation and modulation of adhesion receptors in pediatric patients with congenital heart disease undergoing cardiopulmonary bypass. J Thorac Cardiovasc Surg 1994;107:280-8.

10. Hnatowich DJ, Virzi F, Rusckowski M. Investigations of avidin and biotin for imaging applications. J Nucl Med 1987;28:1294-302.

11. Konijnenberg A, Stokkers EW, van der Post JAM, Schaap MCL, Boer K, Bleker OP, et al. Extensive platelet activation in preeclampsia compared with normal pregnancy: enhanced expression of cell adhesion molecules. Am J Obstet Gynecol 1997;176:461-9.

12. de Haan J, Boonstra PW, Monnink SHJ, Ebels T, van Oeveren W.
Retransfusion of suctioned blood during cardiopulmonary bypass impairs hemostasis. Ann Thorac Surg 1995;59:901-7.

13. Levi M, Cromheecke ME, de Jonge E, Prins MH, de Mol BJM, Briët E, et al. Pharmacological strategies to decrease excessive blood loss in cardiac surgery: a meta-analysis of clinically relevant endpoints. Lancet 1999;354:1940-7.

14. Bizzarri F, Carone E, Caspannini G, Davoli G, Lisi G, Maccherini $\mathrm{M}$, et al. Bleeding reduction in cardiac surgery: a combined approach [letter]. J Thorac Cardiovasc Surg 1998;115:1227-8.

15. Michelson AD, Barnard MR. Plasmin-induced redistribution of platelet glycoprotein Ib. Blood 1990;76:2005-10.

16. Michelson AD, Benoit SE, Kroll MH, Li JM, Rohrer MJ, Kestin AS, et al. The activation-induced decrease in platelet surface expression of the glycoprotein Ib-IX complex is reversible. Blood 1994;83:3562-73.

17. Khan MMH, Gikakis N, Miyamoto S, Rao AK, Cooper SL, Edmunds LH Jr, et al. Aprotinin inhibits thrombin formation and monocyte tissue factor in simulated cardiopulmonary bypass. Ann Thorac Surg 1999;68:473-8. 